Original Research Paper

\title{
Evaluation of Serum Interleukin-8 (CXCL8) Concentrations in Tumor Bearing Dogs
}

\author{
${ }^{1}$ Melanie Haas, ${ }^{2}$ Franz-Josef Kaup and ${ }^{1}$ Stephan Neumann \\ ${ }^{I}$ Tierärztliches Institut, Georg August University of Göttingen, Germany \\ ${ }^{2}$ Pathology Unit, German Primate Center GmbH, Leibniz-Institute for Primate Research, Germany
}

\author{
Article history \\ Received: 13-07-2015 \\ Revised: 24-09-2015 \\ Accepted: 31-10-2015 \\ Corresponding Author: \\ Melanie Haas \\ Tierärztliches Institut, Georg \\ August University of \\ Göttingen, Germany \\ Email: haaster@yahoo.com
}

\begin{abstract}
Interleukin-8 (IL-8 or CXCL8) is a pro-inflammatory and angiogenic chemokine involved in tumorigenesis. Serum CXCL8 is elevated in various neoplastic diseases, but its potential value as a tumor marker in veterinary medicine is unknown. Here, we measured serum CXCL8 concentrations in dogs with various neoplastic diseases using a commercially available canine ELISA kit and analyzed its relationship with patient and disease features. Serum CXCL8 was detected in significantly higher concentrations in tumor bearing dogs compared with healthy patients $(p<0.001)$. Interestingly, serum CXCL8 increased with augmenting tumor size while it decreased with raising malignancy $(p=0.01$ and $p<0.001$, respectively). We found no correlation with the tumor type, tissue origin and histological features such as vascularization, necrosis or infiltrating cells. Some of the associations between CXCL8 and neoplastic features stated in human research were corroborated in this study, suggesting the need for investigating its relevance in tumor bearing dogs.
\end{abstract}

Keywords: CXCL8, Dog, Serum, Tumor

\section{Introduction}

The prevalence of cancer in dogs is rising and remains a major cause of morbidity and mortality (Withrow et al., 2013). Currently available minimallyinvasive methods are insufficient to determine the precise course of disease, making research on blood tumor markers a subject of great importance in veterinary medicine.

In humans it is shown that increased blood and tumoral CXCL8 correlated with poor patient prognosis in a variety of neoplastic processes such as nonHodgkin's lymphoma, melanoma (Nürnberg et al., 1996), breast (Yokoe et al., 1996), colon (Ueda et al., 1994), prostate (Veltri et al., 1999) and ovarian cancer (Yuan et al., 2005) among others. Blocking the angiogenic activity of CXCL8 has already been effective in murine models and the development of humanized monoclonal antibodies has enabled several investigations to determine positive effects when suppressing CXCL8 signaling in human tumors (Heidemann et al., 2003; Waugh and Wilson, 2008; Kim et al., 2014). Even though most studies assure the involvement of CXCL8 in tumorigenesis, the extent of its influence remains controversial (Yao et al., 2007). Some studies report no correlation to angiogenesis and metastatic potential and even attribute CXCL8 a positive role in controlling tumor growth (Yuan et al., 2005; De Larco et al., 2004; Zuccari et al., 2011; Le et al., 2000).

Although there are few studies on CXCL8 in dogs, findings are similar to research in humans and also give contradictory results. An initial study by Zuccari et al. (2011) suggested that CXCL8 plays a protective role in canine mammary cancer since its over-expression was associated with higher survival rates and the absence of metastasis (Zuccari et al., 2011). A later study by the same group had opposite results showing a positive correlation between high serum CXCL8 values and tumor progression, lymph node involvement, metastasis, recurrence and death (Gelaleti et al., 2012). Similarly, a more recent study by De Andrés et al. (2013) supported this finding by assessing local and serum CXCL8 in benign and malignant mammary gland tumors (De Andrés et al., 2013). A study by Kim et al. (2014) on local CXCL8 expression in canine hemangiosarcoma suggested that the effect of CXCL8 is mostly indirect and that its production is part of an adaptive mechanism used by tumor cells to modulate their microenvironment (Kim et al., 2014).

The need to understand tumorigenesis and to improve currently available minimally-invasive diagnostic 
techniques is of great interest to small animal medicine. Serum CXCL8 could aid to predict tumor presence, malignancy and the progression of neoplastic diseases in dogs. Given this, the aim of this study was to determine the potential clinical utility of serum CXCL8 among prevalent neoplastic diseases in dogs by analyzing its behavior in relation to important neoplastic features.

\section{Materials and Methods}

\section{Animals}

In this prospective study 66 client-owned dogs were included from dogs presented between 2011-2014 for clinical evaluation or therapy at the Small Animal Clinic of the University of Göttingen. Inclusion criteria were the presence of a neoplastic disease of any kind and the absence of further tumor unrelated conditions. Enrolled patients underwent a detailed physical examination, complete blood cell (CBC) count and serum chemistry. Case dependent diagnostic procedures were done to diagnose and document the neoplastic disease. As a control group, 23 healthy dogs from the animal shelter "ETN Tierschutzhof Wiesenfeld" in Bad Karlshafen (State of Lower Saxony) were enrolled. Blood samples were taken as part of a routine health examination. The dogs were confirmed to be healthy based on their medical history, detailed physical examination, CBC and serum chemistry. All samples were acquired with the owner's consent and the procedure was in accordance with the German "Protection of Animals Act" and carried out under the supervision of the Commissioner for “Animal Welfare”, Faculty of Agriculture, University of Göttingen.

General, epidemiological and clinical characteristics of the dogs included in this study are listed in Table 1 and 2. The TNM system (size, lymph node involvement, metastasis) established by the World Health Organization (WHO) was taken into consideration whenever given information and group size allowed. Four dogs had an uncertain histological result and were only included in the tests where this information was not required. For every serum CXCL8 sample the information listed in Table 3 was documented and included in the statistical analysis.

\section{Sampling and Analysis Procedures}

\section{Blood Samples}

Blood samples were collected from the cephalic vein previous to any treatment. Blood samples for CBC were collected in polypropylene tubes with $1.6 \mathrm{mg}$ EDTA $/ \mathrm{ml}$ blood (Fa. Sarstedt AG \& Co, Nümbrecht, Germany) and analyzed with a Cell Dyn 3500 Analyzer (Fa. Abbott GmbH \& Co KG, Wiesbaden, Germany). Serum samples required for serum chemistry were collected in standard serum tubes (Fa. Sarstedt AG \& Co, Nümbrecht, Germany) and centrifuged in an Eppendorf centrifuge 5424 (Fa. Eppendorf AG, Hamburg, Germany) at $3000 \times \mathrm{g}$ for five minutes. Serum was extracted from the tube and analyzed according to standardized procedures using a clinical chemistry analyzer (Konelab 20i; Fa. Thermo Fischer Scientific Inc., Dreieich, Germany) and commercial kits.

Table 1. General characteristics of the healthy control dogs and tumor bearing dogs

\begin{tabular}{lll}
\hline & Tumor bearing patients $(\mathrm{n}=66)$ & Healthy control group $(\mathrm{n}=23$ ) \\
\hline Age (in years) & Median $=9.6$ & Median $=5.2$ \\
& Range $=1.9-15.8$ & Range $=1.5-11.5$ \\
& $3-7: \mathrm{n}=16$ & $<3: \mathrm{n}=3$ \\
& $>7: \mathrm{n}=49$ & $3-7: \mathrm{n}=12$ \\
& & $>7: \mathrm{n}=4$ \\
Gender & Female $\mathrm{n}=41$ & Female $\mathrm{n}=5$ \\
& Neutered females $\mathrm{n}=11$ & Neutered females $\mathrm{n}=4$ \\
& Male $\mathrm{n}=25$ & Male $\mathrm{n}=18$ \\
& Neutered males $\mathrm{n}=9$ & Neutered males $\mathrm{n}=13$ \\
& Mixed $\mathrm{n}=21$ & Mixed $\mathrm{n}=20$ \\
& Golden Retriever $\mathrm{n}=5$ & German Shepherd $\mathrm{n}=2$ \\
& Bavarian Mountain Hound $\mathrm{n}=4$ & Boxer $\mathrm{n}=1$ \\
& Teckel $\mathrm{n}=4$ & \\
& Australian Shepherd $\mathrm{n}=2$ & \\
& Hovawart $\mathrm{n}=2$ & \\
& Old German Herding Dog $\mathrm{n}=2$ & \\
& Bernese Mountain Dog $\mathrm{n}=2$ & \\
& Cocker Spaniel $\mathrm{n}=2$ & \\
German Shepherd $\mathrm{n}=2$ & Airdale Terrier $\mathrm{n}=2$ & \\
Dalmatian $\mathrm{n}=2$ & \\
Labrador Retriever $\mathrm{n}=2$ & \\
Other breeds $\mathrm{n}=14$ & \\
&
\end{tabular}


CXCL8 was measured in serum since preliminary results showed a higher reliability than plasma values (data not shown). Serum samples used to determine the CXCL8 concentrations were processed following the R\&D Systems protocol. Samples were kept at room temperature for two hours to allow clotting and were then centrifuged for $20 \mathrm{~min}$ at $1000 \times \mathrm{g}$. Serum was immediately aliquoted with a Pipetman P100 (Fa. Gilson, Villers Le Bel, France) and stored at $-20^{\circ} \mathrm{C}$ until required for analysis. Repeated freeze-thaw cycles were avoided.

\section{Assessment of CXCL8}

Serum CXCL8 levels were measured in triplicate using a commercially available canine CXCL8 quantitative ELISA kit (Quantikine, R\&D Systems, Minneapolis, MN, USA) following the manufacturer's instructions. The optical density of each well was determined within 30 min using a TECAN microplate reader at an optical density of $450 \mathrm{~nm}$ and a wavelength correction of $570 \mathrm{~nm}$. Calibration was performed using a standard series of dilutions of recombinant canine CXCL8 provided in the kit. The CXCL8 concentration $(\mathrm{pg} / \mathrm{ml})$ of each sample was calculated through a data analysis software (Magellan, Fa. TECAN GmbH, Grödig, Austria). According to the manufacturer's instructions, the assay measures the natural and recombinant CXCL8 isoforms of 74 and 79 amino acids and the minimum detectable dose of CXCL8 is $1.26 \mathrm{pg}$ $\mathrm{mL}$. The assay shows no cross-reactivity with a series of cytokines and growth factors. The manufacturer reported mean intra-and inter-assay coefficients of variation of 5.6 and $5.3 \%$, respectively.

\section{Histology}

Histological samples were obtained through surgical tumor excision or Tru-Cut biopsies. Samples were fixed in $10 \%$ buffered formalin, routinely embedded in paraffin and stained with hematoxylin and eosin. Histopathological examinations were performed by a board-certified pathologist (European College of Veterinary Pathologists, ECVP) and classified according to the WHO histological classification. Information on the involvement of inflammatory cells, vascularization, bleedings, necrosis and infiltration of adjoining tissue was used to estimate the tumor malignancy and documented for statistical analysis on its correlation with serum CXCL8 values.

\section{Statistical Analysis}

Statistical analysis was performed using a commercial software (MATLAB and Statistics Toolbox Release 2014a, The Math Works, Inc., Natick, Massachusetts, United States). CXCL8 concentrations were grouped according to goal criteria and the nullhypothesis that two groups came from the same population was tested with a non parametric MannWhitney-U-Test. Additionally a link between CXCL8 and numeric parameters (age, blood values and onset of disease) was determined by the Pearson's correlation coefficient and Spearman's rank correlation coefficient. To find a threshold serum CXCL8 value that maximizes the discrimination power between two groups a Receiver Operating Characteristic analysis (ROC) was used. Since epidemiological and clinical characteristics are given in ordinal categorical classes, the relation between the serum CXCL8 concentration and increase in category was analyzed using Generalized Linear Models (GLM). For this, CXCL8 values were analyzed $\log$ transformed and both process and observation error were assumed to have Gaussian distributions. A $p$ value below 0.05 value was considered statistically significant.

\section{Results}

Through the statistical evaluation of the general characteristics (Table 1) and the documented information (Table 3) an influence of these features on serum CXCL8 concentrations was ruled out. This allowed as a first approach to join the patients into groups according to epidemiological and clinical characteristics (Table 2) without taking general information into account.

Table 2. Epidemiological and clinical characteristics of tumor bearing dogs

\begin{tabular}{llrr}
\hline & & $\mathrm{n}=66$ & \multicolumn{1}{c}{$\%$} \\
\hline Malignancy & Benign & 10 & 15.15 \\
& Low malignant & 10 & 15.15 \\
& Malignant & 35 & 53.03 \\
& Highly malignant & 7 & 10.61 \\
& Unknown & 4 & 6.06 \\
Tumor type & Carcinoma & 20 & 30.30 \\
& Sarcoma & 8 & 12.12 \\
& Lymphoma & 5 & 7.58 \\
& Mastocytoma & 5 & 7.58 \\
Tissue of origin & Malignant Histiocytosis & 1 & 1.52 \\
& Mixed Tumors & 3 & 4.55 \\
& Benign Tumors & 12 & 18.18 \\
& Other tumor types & 13 & 19.70 \\
& Eesithelial & 26 & 39.39 \\
& Lymphochymal & 17 & 25.76 \\
& Other tissues of origin & 5 & 7.58 \\
Affected organ & 18 & 27.27 \\
& Mammary gland & 27 & 40.91 \\
& Skin & 6 & 9.09 \\
& Spleen & 6 & 9.09 \\
& Liver & 7 & 10.61 \\
& Other organs & 20 & 30.30 \\
& $<2$ cm & 14 & 21.21 \\
& 2-4 cm & 14 & 21.21 \\
& Tumor size & 30 & 45.45 \\
& Unknown/Diffuse & 3 & 4.55 \\
\hline & & &
\end{tabular}


The serum CXCL8 levels of tumor bearing dogs ranged from $109 \mathrm{pg} \mathrm{mL}$ to $7611.2 \mathrm{pg} \mathrm{mL}$, with a median of $1750.4 \mathrm{pg} \mathrm{mL}$ (interquartile range: 1307.1-2748.8 pg $\mathrm{mL}$ ). The average level in these patients was significantly higher $(\mathrm{p}<0.001)$ than that of healthy controls (Range: 383.79-2213.3, median: $946.91 \mathrm{pg} \mathrm{mL}$, interquartile range: 671.67-1131.6 pg $\mathrm{mL}$ ) (Fig. 1a). This difference was significant regardless of the size of the tumor $(<2 \mathrm{~cm}$ $\mathrm{p}=0.007,>2 \mathrm{~cm} \mathrm{p}<0.001)$ or if the tumor was benign $(\mathrm{p}=$ $0.007)$ or malignant $(p<0.001)$ (Fig. $1 c-d)$. A CXCL8 cutoff value to discriminate healthy from tumor bearing dogs was calculated using a ROC analysis. A value of 1196.8 $\mathrm{pg} / \mathrm{ml}$ optimized specificity at $91 \%$ and sensitivity at $78 \%$ (AUC $=0.8154)$ (Fig. 1b).

Patients with tumors larger than $4 \mathrm{~cm}$ had significantly higher values than those with tumors of less than $2 \mathrm{~cm}(\mathrm{p}=0.047)$ (Fig. 2a). Small $(<2 \mathrm{~cm})$ and medium $(2-4 \mathrm{~cm})$ sized tumors could not be statistically distinguished from each other $(\mathrm{p}=0.90)$, but a GLM showed an association between an increase in the size category and a serum CXCL8 raise $(p=0.01)$ (Fig. $2 b)$.

The median measurement of serum CXCL8 decreased with increasing tumor malignancy, but the categories were not statistically different from each other (benign-low $\mathrm{p}=0.67$, benign-malignant $\mathrm{p}=0.52$, benign-high $\mathrm{p}=0.18$, low-malignant $\mathrm{p}=0.67$, low-high $\mathrm{p}=0.11$, malignant-high $\mathrm{p}=0.09$ ) (Fig. 3a). Interestingly, highly malignant tumors were indistinguishable from healthy dogs $(\mathrm{p}=0.96)$ (Fig. 3b).

Based on this trend a GLM relating serum CXCL8 with tumor malignancy was employed without a significant result $(p=0.1)$. Since healthy controls were indistinguishable from dogs with highly malignant tumors, this group was included as an additional malignancy category. In this case a GLM showed an association between an increase in the malignancy category (benign $>$ low $>$ malignant $>$ highly $>$ healthy) and a decrease of serum CXCL8 ( $<<0.001)$ (Fig. 3c).

Serum CXCL8 concentrations were not predictive of the tumor type and a distinction was still not possible when size and malignancy were taken into account by using GLM (data not shown). Only carcinomas and lymphomas appeared to be significantly different from each other $(p=0.03)$ (Fig. 4a). To test if this difference was caused by the tumor size, since there was an increased number of large lymphomas, carcinomas were split into two groups according to the tumor size $(<4 \mathrm{~cm}$ $(\mathrm{n}=9)$ and $>4 \mathrm{~cm}(\mathrm{n}=9))$. This analysis showed that only carcinomas $<4 \mathrm{~cm}$ were significantly different from lymphomas $(<4 \mathrm{~cm} \mathrm{p}=0.02,>4 \mathrm{~cm} \mathrm{p}=0.23)$ (Fig. $4 \mathrm{~b})$.

Table 3. Documented information

Animals general condition
CBC and serum chemistry
Onset of disease
Amount of tumors
Histological characteristics
(of the tumor)

\section{Good \\ Moderate poor \\ Numeric values}

In days

Numeric values

Inflammatory cells

Vascularization

Bleedings

Necrosis

Tissue invasion

Prognosis

Good

Guarded

Poor

Remission

No change

Recurrence

Death-euthanasia
Low

Normal

Mildly increased

Highly increased

Lymphoplasmatic Mononuclear

Mixed

No

Low-moderate

High

No

Low-moderate

High

No

Low-moderate

High

Lymphatic vessels

Conective tissue

Intraductal

Adipose tissue

Blood vessels

Multiple 
a

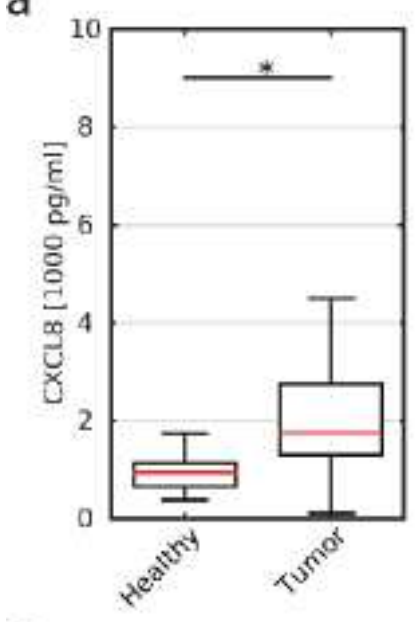

C

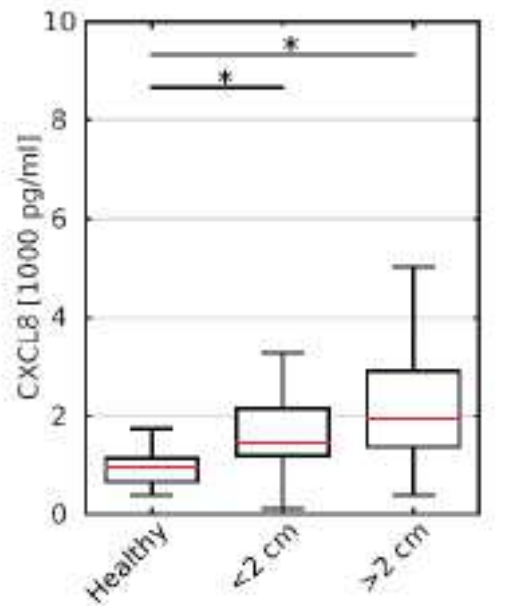

b

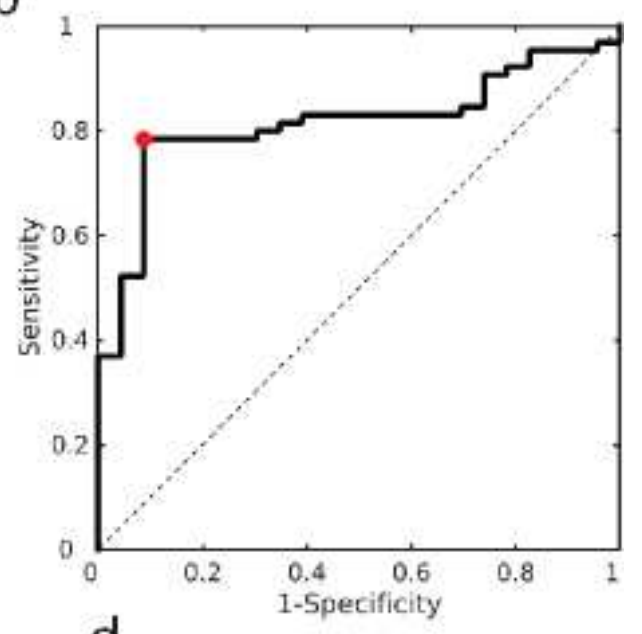

d

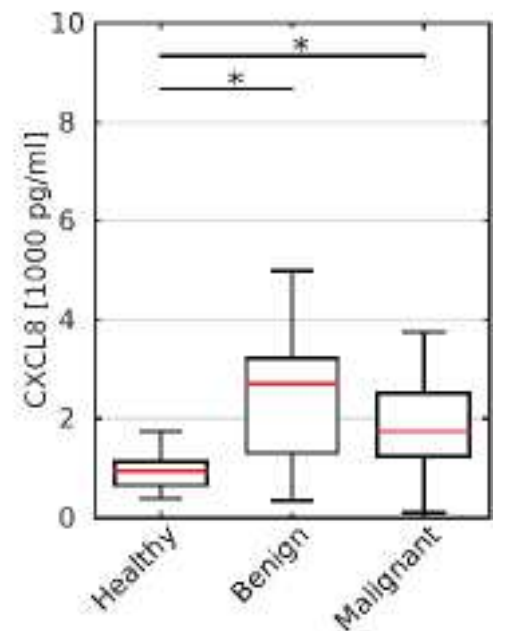

Fig. 1. Tumor bearing patients have higher serum CXCL8 values than healthy dogs. (a) Comparison of the serum CXCL8 levels among the healthy control group and tumor bearing patients showing a significant difference $(\mathrm{p}<0.001)$. (b) Receiver operating characteristic analysis of the healthy control group and tumor bearing patients. A discrimination of the groups can be made at a CXCL8 cutoff value of $1196.8 \mathrm{pg} / \mathrm{mL}$ with a specificity of $91 \%$ and a sensitivity of $78 \%$ (AUC $=0.8154)$. c-d) Comparison of the healthy control group and tumor bearing dogs grouped in tumor size $<2 \mathrm{~cm}$ and $>2 \mathrm{~cm}$ (c) and benign and malignant tumors (d), showing that the difference between healthy and tumor bearing patients is significantly independent of tumor size and malignancy

a

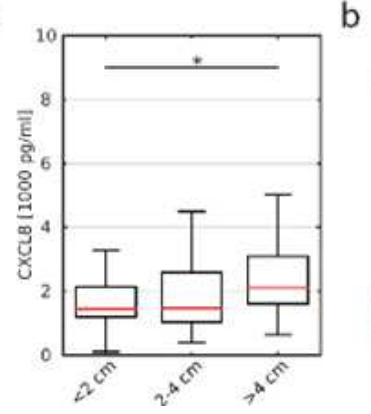

b
Estimated Coefficients:

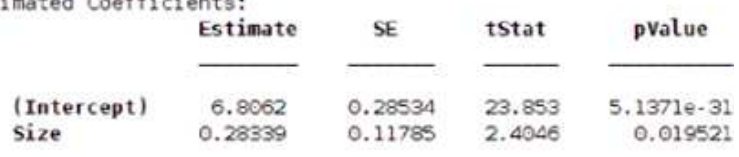

58 observations, 56 error degrees of freedor

Estimated Dispersion: 0.55

F.statistic vs. constant model: $5.78, p$-value $=0.0195$

Fig. 2. An increase in tumor size is associated with rising concentrations of CXCL8 in serum. (a) Comparison of serum CXCL8 concentrations among different tumor sizes. (b) Generalized linear model $\log$ (CXCL8) $\sim 1+$ tumor size shows a significant positive relationship 
a

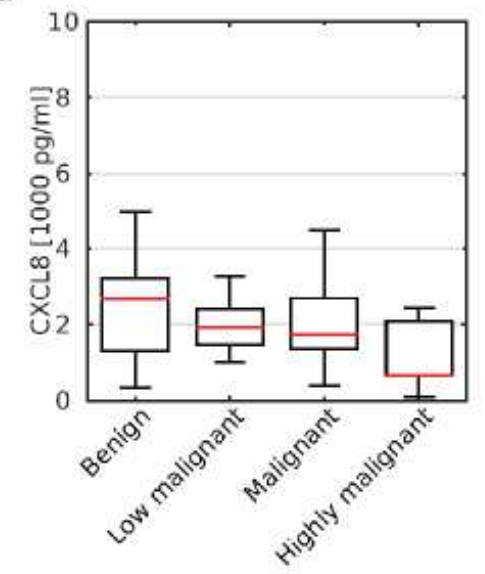

c

Estimated Coefficients:

$$
\begin{aligned}
& \text { - } \\
& \text { (Intercept) } \\
& 7.8499 \\
& -0.2527 \\
& \text { 0. } 15402 \\
& 0.058913
\end{aligned}
$$

b

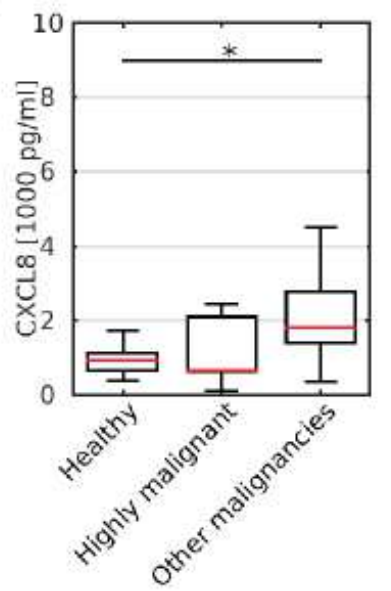

Fig. 3. An increase in tumor malignancy is associated with a decrease in serum CXCL8 levels. (a) Comparison of serum CXCL8 among the different categories of malignancy with no significant difference between the groups. (b) Comparison of serum CXCL8 between the healthy control group, highly malignant and a group consisting of categories lower than highly malignant tumors. Highly malignant tumors are not significantly different from healthy patients (c) Generalized linear model log (CXCL8) $1+$ malignancy (including the healthy control groups as a malignancy category) shows a significant negative relationship

a

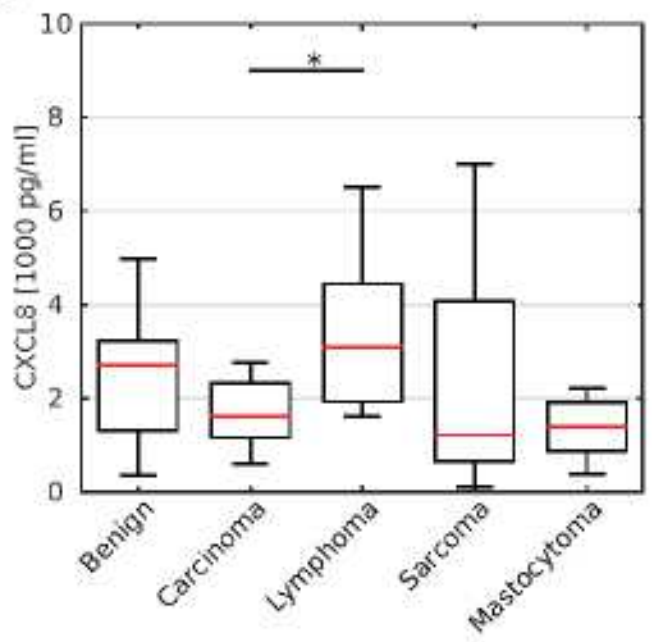

b

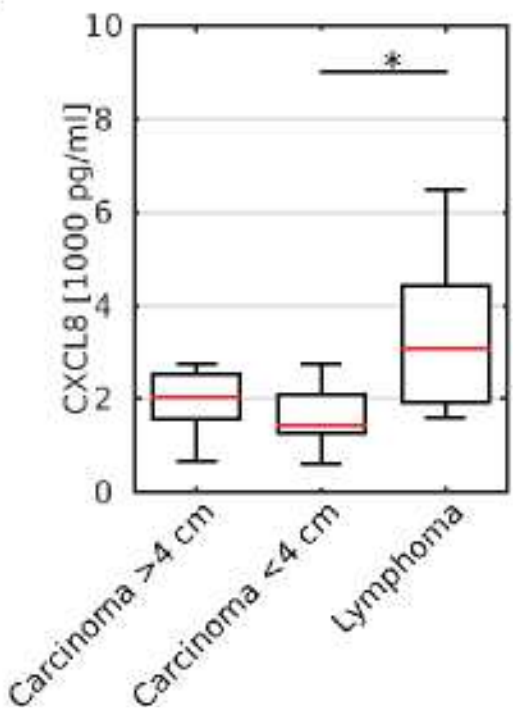

Fig. 4. Serum CXCL8 concentrations were not predictive of the tumor type. (a) Comparison of serum CXCL8 among the most common tumor types of the sample set. Only carcinoma and lymphoma were statistically different from each other $(\mathrm{p}=0.03)(\mathrm{b})$ Comparison of lymphoma and carcinomas grouped in tumor size $<4 \mathrm{~cm}$ and $>4 \mathrm{~cm}$. A significant difference between carcinoma and lymphoma is size dependent 
Serum CXCL8 concentrations were not predictive of the tissue origin (Epithelial-mesenchymal $\mathrm{p}=0.56$, epithelial-lymphocytic $\mathrm{p}=0.07$, mesenchymallymphocytic $\mathrm{p}=0.13$ ). This distinction was also not possible when size and malignancy information was taken into account by using GLM (Data not shown).

Finally, serum CXCL8 was statistically unrelated to local neoplastic properties such as presence and grade of inflammatory cells, necrosis, vascularization and bleedings (Data not shown).

\section{Discussion}

Tumor development is a complex process dependent on multiple factors and interactions. Several studies affirm that the highly selective pro-inflammatory and angiogenic chemokine Interleukin-8 (IL-8 or CXCL8) is involved in this process and therefore, is a potential tumor marker (Yuan et al., 2005; Kim et al., 2014; Raman et al., 2007; Strieter et al., 2004; Koch et al., 1992; Baggiolini et al., 1989; Addison et al., 2000). However, research into CXCL8 in veterinary medicine is scarce.

We found that tumor bearing patients have higher serum CXCL8 values than healthy dogs. Although the major production of CXCL8 occurs locally as an adaptive response of cancer cells to withstand environmental or chemical stresses (Kim et al., 2014; Raman et al., 2007; Desbaillets et al., 1997; Shi et al., 2001), its concentration has been found to be also elevated in the blood of human and canine cancer patients (Yokoe et al., 1996; Ueda et al., 1994; Veltri et al., 1999; Gelaleti et al., 2012; De Andrés et al., 2013; Konno et al., 2003; Ugurel et al., 2001; Ren et al., 2003; Benoy et al., 2004; Lokshin et al., 2006). On the one hand, cells within the tumor tissue are known to secrete CXCL8 that may partly escape the tumor environment and reach the bloodstream through the vast amount of blood vessels that usually nourish a tumor. On the other hand, in healthy individuals and in patients with autoimmune or inflammatory diseases, CXCL8 is produced systemically by inflammatory cells and cannot be excluded in tumor bearing patients as an attempt to fight the abnormal neoplastic tissue. Yet, serum CXCL8 seems to reflect local neoplastic changes.

In this study a significant correlation between augmented serum CXCL8 concentrations and an increasing tumor size was found, being consistent with previous studies (Konno et al., 2003; Ren et al., 2003; Ahmed et al., 2006). Locally, CXCL8 is known to moderate tumor growth and survival through neovascularization, as well as autocrine and paracrine mechanisms, but the exact mechanism for its raise in peripheral blood is unknown (Yuan et al., 2005; De Larco et al., 2004; Raman et al., 2007; Balkwill, 2003). A possible explanation is that large tumors are comprised of more cells that secrete CXCL8. Moreover, large tumors commonly have a higher inflammatory, hemorrhagic and necrotic component which elicits a stronger CXCL8 expression (Raman et al., 2007). Since larger tumors also have a more pronounced vasculature this would facilitate CXCL8 to enter the bloodstream. Still, an augmented systemic production of CXCL8 in response to the progressing tumor growth and inflammation cannot be discarded.

Our study revealed that even though tumor bearing patients have higher values than healthy patients, serum CXCL8 decreased with increasing malignancy. A clear statistical distinction of the stages of malignancy could not be made but a negative relation was observable through GLM. Higher malignancy would be rather expected to be associated with an increase of CXCL8 in peripheral blood due to its mechanisms of action that create a favorable environment for tumor development (Heidemann et al., 2003; Raman et al., 2007; Addison et al., 2000; Schruefer et al., 2005). Nevertheless, previous research on CXCL8 in peripheral blood has been controversial. Many studies have shown, that elevated CXCL8 in sera was associated with higher malignancy and an advanced stage of disease in breast cancer (Yokoe et al., 1996; Benoy et al., 2004), melanoma (Ugurel et al., 2001), hepatocellular carcinoma (Ren et al., 2003) and soft tissue sarcoma (Rutkowski et al., 2002). Other studies did not find any correlation or suggested that CXCL8 could play a protective role in cancer as it was diminished in highly malignant tumors (Yuan et al., 2005; De Larco et al., 2004; Zuccari et al., 2011; Le et al., 2000; Derin et al., 2007). However, in contrast to our study the relationship between CXCL8 and tumor malignancy has mostly been analyzed in specific tumor types. A comparative evaluation of CXCL8 among different tumor types including benign masses has rarely been done. Thus, these are novel results that should be further investigated.

Interestingly, the results of our study showed that patients with benign masses had the highest serum CXCL8 values among all malignancy groups and were therefore significantly different from healthy dogs. It has been reported that serum CXCL8 values are also increased in benign masses, but these levels have not been compared to that of malignant tumors (Tamura et al., 2013). Although it is known that most tumor cells produce CXCL8, the ability of these cells to express and release CXCL8 depends on a proper stimulus that might not be given with increasing malignancy (Baggiolini et al., 1989). Another possible reason is that the malignancyrelated local CXCL8 increase leads to a blockage of its systemic production through a negative feedback mechanism. So far, such a mechanism has not been described. Additionally, serum CXCL8 is susceptible to changes in its concentration due to dilution and its reliability to predict local changes should therefore be questioned. Although some studies showed a congruent increase in local and systemic CXCL8 values (De Andrés et al., 2013; Tamura et al., 2013) a study by Konno et al. 
(2003) found a relationship between CXCL8 and a variety of tumor parameters in drainage veins of gastric cancer, whereas no significant relationship in peripheral veins (Konno et al., 2003). Moreover, the producing cells as well as the function of local and systemic CXCL8 may differ so that both should be included and compared in future studies.

In our study serum CXCL8 concentrations were not predictive of the tumor type or the tissue origin and was similarly altered regardless of the tumor vascularization, tumor necrosis and leucocytes infiltrating the tumor tissue. CXCL8 is predominantly produced by leucocytes and endothelial cells (Raman et al., 2007; Strieter et al., 2004; Baggiolini et al., 1989) and therefore controls leukocyte infiltrate, tumor immune response and neovascularization. Yet, serum CXCL8 has only been studied separately in various tumor types and information about its correlation with these characteristics is limited (Konno et al., 2003; Ugurel et al., 2001; Ren et al., 2003; Benoy et al., 2004; Lokshin et al., 2006; Ahmed et al., 2006; Balkwill, 2003; Schruefer et al., 2005; Rutkowski et al., 2002; Derin et al., 2007; Tamura et al., 2013; Scheibenbogen et al., 1995).

The lack of statistical significance of certain comparisons may be due to the low sample size of some categories and thus larger investigations are warranted. Moreover, it is important to analyze which cells are responsible for the serum CXCL8 increase in tumor bearing dogs to allow detailed research that clarifies the true influence and predictive potential of serum CXCL8 in tumorigenesis.

\section{Conclusion}

The secretion of CXCL8 seems not be characteristic of a determined neoplastic disease but a common event in most tumor patients. Augmented serum CXCL8 concentrations are dependent on the tumor size and its malignancy. Although the predictive value of serum CXCL8 in neoplastic diseases was insufficient, this study supports that CXCL8 is involved in the pathogenesis of neoplastic diseases in dogs. Thus, the extent of its involvement, as well as the exact mechanisms and functions of this chemokine in tumorigenesis, should be further analyzed in veterinary medicine.

\section{Acknowledgment}

We thank Juan Daniel Flórez Weidinger (Max Planck Institute for Dynamics and Self-Organisation) for support on statistical analyses. We thank Rafael Polania, Silvia Hennecke and Carmen Murall for discussions.

\section{Funding Information}

This research was funded by the Tierärztliches Institut Göttingen, Germany.

\section{Author's Contributions}

Melanie Haas: Study desigen sampling, sample processing, statistical analysis and preparation of the manuscript.

Franz-Josef Kaup: Histopathological evaluation of neoplastic tissue and preparatiion of the manuscript.

Stephan Neumann: Study design and preparation of the manuscript.

\section{Ethics}

This article is original and contains unpublished material. The corresponding author confirms that all of the other authors have read and approved the manuscript and no ethical issues involved.

\section{References}

Addison, C.L., T.O. Daniel, M.D. Burdick, H. Liu and J.E. Ehlert et al., 2000. The CXC chemokine receptor 2, CXCR2, is the putative receptor for ELR + CXC chemokine-induced angiogenic activity. J. Immunol., 165: 5269-77. DOI: 10.4049/jimmunol.165.9.5269

Ahmed, O.I., A. Adel, D. Diab and N. Gobran, 2006. Prognostic value of serum level of interleukin- 6 and interleukin-8 in metastatic breast cancer patients. Egyptian J. Immunol., 13: 61-68. PMID: 18689272

Baggiolini, M., A. Walz and S.L. Kunkel, 1989. Neutrophil-activating peptide-1/interleukin 8 , a novel cytokine that activates neutrophils. J. Clin. Investigat., 84: 1045-1049. DOI: 10.1172/JCI114265

Balkwill, F., 2003. Chemokine biology in cancer. Seminars Immunol., 15: 49-55. DOI: $10.1016 / \mathrm{S} 1044-5323(02) 00127-6$

Benoy, I.H., R. Salgado, P.V. Dam, K. Geboers and E.V. Marck et al., 2004. Increased serum interleukin-8 in patients with early and metastatic breast cancer correlates with early dissemination and survival. Clin. Cancer Res., 10: 7157-7162. DOI: 10.1158/1078-0432.CCR-04-0812

De Andrés, P.J., J.C. Illera, S. Cáceres, L. Díez and M.D. Pérez-Alenza et al., 2013. Increased levels of interleukins 8 and 10 as findings of canine inflammatory mammary cancer. Vet. Immunol. Immunopathol., 152: 245-251. DOI: $10.1016 /$ j.vetimm.2012.12.010

De Larco, J.E., B.R. Wuertz and L.T. Furcht, 2004. The potential role of neutrophils in promoting the metastatic phenotype of tumors releasing interleukin-8. Clin. Cancer Res., 10: 4895-4900. DOI: 10.1158/1078-0432.CCR-03-0760

Derin, D., H.O. Soydinc, N. Guney, F. Tas and H. Camlica et al., 2007. Serum IL-8 and IL-12 levels in breast cancer. Med. Oncol., 24: 163-168. DOI: $10.1007 / \mathrm{BF} 02698035$ 
Desbaillets, I., N. de Tribolet, M.F. Hamou and E.G. Van Meir, 1997. Upregulation of interleukin 8 by oxygendeprived cells in glioblastoma suggests a role in leukocyte activation, chemotaxis and angiogenesis. J. Experimental Med., 186: 1201-1212.

DOI: $10.1084 /$ jem.186.8.1201

Gelaleti, G.B., B.V. Jardim, C. Leonel, M.G. Moschetta and D. Zuccari, 2012. Interleukin-8 as a prognostic serum marker in canine mammary gland neoplasias. Vet. Immunol. Immunopathol., 146: 106-112. DOI: 10.1016/j.vetimm.2012.02.005

Heidemann, J., H. Ogawa, M.B. Dwinell, P. Rafiee and C. Maaser et al., 2003. Angiogenic effects of interleukin 8 (CXCL8) in human intestinal microvascular endothelial cells are mediated by CXCR2. J. Biol. Chem., 278: 8508-8515. DOI: $10.1074 /$ jbc.M208231200

Kim, J.H., A.M. Frantz, K.L. Anderson, A.J. Graef and M.C. Scott et al., 2014. Interleukin-8 promotes canine hemangiosarcoma growth by regulating the tumor microenvironment. Experimental Cell Res., 323: 155-164. DOI: 10.1016/j.yexcr.2014.02.020

Koch, A.E., P.J. Polverini, S.L. Kunkel, L.A. Harlow and L.A. DiPietro et al., 1992. Interleukin-8 as a macrophage-derived mediator of angiogenesis. Science, 258: 1798-1801.

DOI: $10.1126 /$ science. 1281554

Konno, H., M. Ohta, M. Baba, S. Suzuki and S. Nakamura, 2003. The role of circulating IL-8 and VEGF protein in the progression of gastric cancer. Cancer Sci., 94: 735-740. DOI: 10.1111/j.1349-7006.2003.tb01511.x

Le, X., Q. Shi, B. Wang, Q. Xiong and C. Qian et al., 2000. Molecular regulation of constitutive expression of interleukin- 8 in human pancreatic adenocarcinoma. J. Interferon Cytokine Res., 20: 935-946. DOI: $10.1089 / 10799900050198372$

Lokshin, A.E., M. Winans, D. Landsittel, A.M. Marrangoni and L. Velikokhatnaya et al., 2006. Circulating IL-8 and anti-IL- 8 autoantibody in patients with ovarian cancer. Gynecol. Oncol., 102: 244-251. DOI: 10.1016/j.ygyno.2005.12.011

Nürnberg, W., D. Tobias, F. Otto, B.M. Henz and D. Schadendorf, 1996. Expression of interleukin-8 detected by in situ hybridization correlates with worse prognosis in primary cutaneous melanoma. J. Pathol., 189: 546-551.

DOI: $10.1002 /($ SICI)1096-

9896(199912)189:4<546::AID-

PATH487>3.0.CO;2-L

Raman, D., P.J. Baugher, Y.M. Thu and A. Richmond, 2007. Role of chemokines in tumor growth. Cancer Lett., 256: 137-165.

DOI: $10.1016 /$ j.canlet.2007.05.013
Ren, Y., R.T. Poon, H. Tsui, W.H. Chen and Z. Li et al., 2003. Interleukin- 8 serum levels in patients with hepatocellular carcinoma: Correlations with clinicopathological features and prognosis. Clin. Cancer Res., 9: 5996-6001. PMID: 14676125

Rutkowski, P., J. Kaminska, M. Kowalska, W. Ruka and J. Steffen, 2002. Cytokine serum levels in soft tissue sarcoma patients: Correlations with clinicopathological features and prognosis. Int. J. Cancer, 100: 463-471. DOI: 10.1002/ijc. 10496

Scheibenbogen, C., T. Möhler, J. Haefele, W. Hunstein and U. Keilholz, 1995. Serum interleukin-8 (IL-8) is elevated in patients with metastatic melanoma and correlates with tumour load. Melanoma Res., 5: 139-195.

Schruefer, R., N. Lutze, J. Schymeinsky and B. Walzog, 2005. Human neutrophils promote angiogenesis by a paracrine feedforward mechanism involving endothelial interleukin-8. Am. J. Physiol. Heart Circulatory Physiol., 288: H1186-H1192. DOI: 10.1152/ajpheart.00237.2004

Shi, Q., Q. Xiong, X. Le and K. Xie, 2001. Regulation of interleukin-8 expression by tumor-associated stress factors. J. Interferon Cytokine Res., 21: 553-566. DOI: 10.1089/10799900152547812

Strieter, R.M., J.A. Belperio, R.J. Phillips and M.P. Keane, 2004. CXC chemokines in angiogenesis of cancer. Seminars Cancer Biol., 14: 195-200. DOI: 10.1016/j.semcancer.2003.10.006

Tamura, Y., H. Ohta, S. Torisu, M. Yuki and N. Yokoyama et al., 2013. Markedly increased expression of interleukin-8 in the colorectal mucosa of inflammatory colorectal polyps in miniature dachshunds. Vet. Immunol. Immunopathol., 156: 32-42. DOI: $10.1016 /$ j.vetimm.2013.09.017

Ueda, T., E. Shimada and T. Urakawa, 1994. Serum levels of cytokines in patients with colorectal cancer: possible involvement of interleukin-6 and interleukin8 in hematogenous metastasis. J. Gastroenterol., 29: 423-429. DOI: $10.1007 / \mathrm{BF} 02361238$

Ugurel, S., G. Rappl, W. Tilgen and U. Reinhold, 2001. Increased serum concentration of angiogenic factors in malignant melanoma patients correlates with tumor progression and survival. J. Clin. Oncol., 19: 577-583. PMID: 11208853

Veltri, R.W., M.C. Miller, G. Zhao, A. Ng and G.M. Marley et al., 1999. Interleukin-8 serum levels in patients with benign prostatic hyperplasia and prostate cancer. Urology, 53: 139147. DOI: 10.1016/S0090-4295(98)00455-5

Waugh, D.J. and C. Wilson, 2008. The interleukin-8 pathway in cancer. Clin. Cancer Res., 14: 6735-6741. DOI: 10.1158/1078-0432.CCR-07-4843

Withrow, S.J., D.M. Vail and R. Page, 2013. Withrow and MacEwen's Small Animal Clinical Oncology. 5th Edn., Elsevier Health Sciences, St. Louis, ISBN-10: 1437723624, pp: 750. 
Yao, C., Y. Lin, MS. Chua, C.S. Ye and J. Bi et al., 2007. Interleukin-8 modulates growth and invasiveness of estrogen receptor-negative breast cancer cells. Int. J. Cancer, 121: 1949-1957. DOI: $10.1002 /$ ijc. 22930

Yokoe, T., Y. Iino, H. Takei, J. Horiguchi and Y. Koibuchi et al., 1996. Changes of cytokines and thyroid function in patients with recurrent breast cancer. Anticancer Res., 17: 695699. PMID: 9066604
Yuan, A., J.J. Chen, P.L. Yao and PC. Yang, 2005. The role of interleukin-8 in cancer cells and microenvironment interaction. Frontiers Biosci., 10: 853-865. PMID: 15569594

Zuccari, D.A.PC., R. Castro, G.B. Gelaleti and U.M. Mancini, 2011. Interleukin-8 expression associated with canine mammary tumors. Genet. Mol. Res., 10: 1522-1532. DOI: 10.4238/vol10-3gmr1145 